\title{
Time lags between starburst and AGN activity in galaxy mergers
}

\author{
M. Blank ${ }^{1,3}$ and W. J. Duschl ${ }^{1,2,4}$ \\ ${ }^{1}$ Institut für Theoretische Physik und Astrophysik, \\ Christian-Albrechts-Universität zu Kiel, Germany \\ ${ }^{2}$ Steward Observatory, The University of Arizona, Tucson, AZ, USA \\ ${ }^{3}$ mblank@astrophysik.uni-kiel.de \\ ${ }^{4}$ wjd@astrophysik.uni-kiel.de
}

\begin{abstract}
We show that the observed time lag between starburst and AGN activity can be explained by a viscous time lag the gas needs to flow through the AGN's accretion disk before reaching the central black hole. Our calculations reproduce the observed time lag and are in agreement with the observed correlation between black hole mass and stellar velocity dispersion.
\end{abstract}

Keywords. galaxies: active — galaxies: interactions — galaxies: nuclei — galaxies: starburst

\section{Introduction}

Recent observations of active galaxies show that AGN activity may be delayed with regard to star formation activity by time scales of 50-250 Myr (e.g., Wild et al. 2010). We explain this time lag by modeling the inflow of mass towards the central black hole in the framework of an accretion disk scenario. Thus the time lag between starburst and AGN activity principally consists of a viscous lag the gas needs to flow through the accretion disk until it reaches the black hole. In our model setup two gas rich disk galaxies are on a parabolic collision course and then merge, forming a gas poor elliptical galaxy. The merger causes a starburst and the inflow of gas towards the center of the newly forming galaxy. Before reaching the center, the gas first loses angular momentum due to gravitational instabilities. Some 100 parsecs from the center it forms an accretion disk and loses further angular momentum due to viscous torques. The gas finally reaches the black hole, leading to the activity of the galactic nucleus.

\section{Numerical methods}

We simulate galaxy collisions using the TreeSPH code GADGET-2 (Springel 2005). We include star formation as described by Scannapieco et al. (2005) and mechanical AGN feedback following Debuhr et al. (2012). The AGN is represented by an accretion disk particle (ADP, Power et al. 2011) that accretes gas particles from its environment. The black hole growth rate is calculated via a subgrid model: The ADP contains a black hole and an accretion disk, the mass accreted by the ADP is added to the outer rim of the accretion disk, from where it is accreted towards the black hole on a viscous time scale.

\section{Results}

The merging event drives huge amounts of gas towards the cent-re of the newly forming galaxy where it causes a starburst with a lifetime of $200-300 \mathrm{Myr}$ and feeds the AGN as shown in Figure 1. After being devoured by the AGN the gas flows through the 

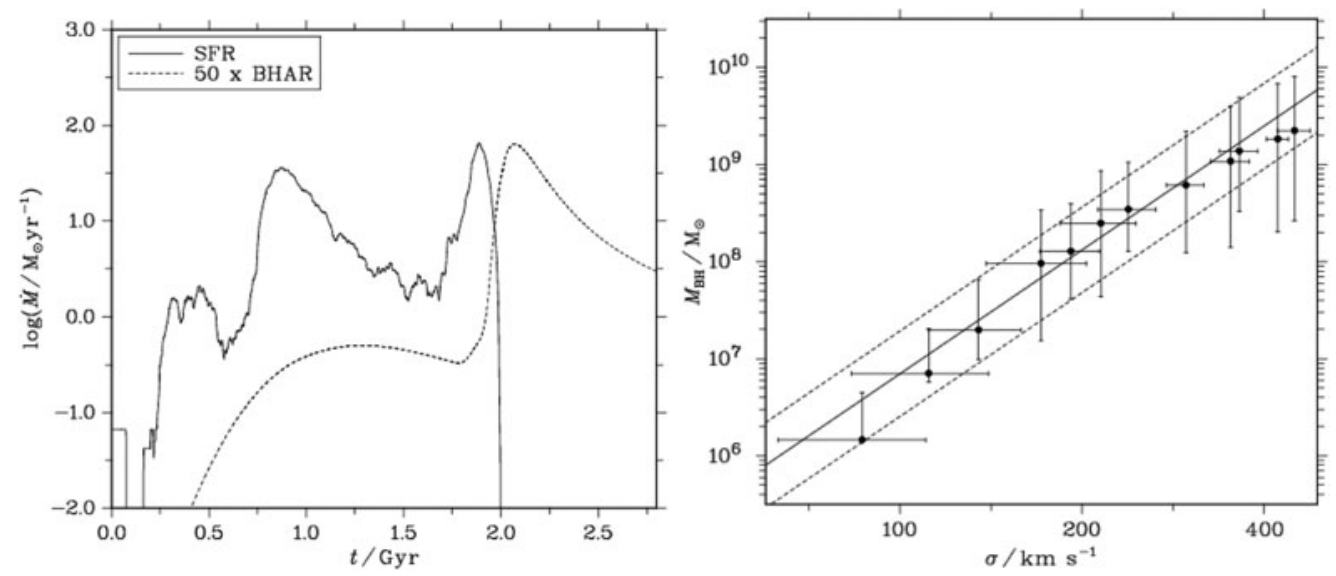

Figure 1. Star formation rate and black hole Figure 2. Dots: black hole mass after the accretion rate as functions of time. The two merging event. Horizontal bars: error of $\sigma$. Vermerging galaxies have approximately the mass tical bars: range of black hole mass during its and the size of the Milky Way. growth stage. Solid line: observed $M_{\mathrm{BP}}-\sigma$ correlation with intrinsic scatter (dashed lines) according to Gültekin et al. (2009).

accretion disk on a viscous timescale that corresponds to a time lag of approximately 200 Myr between the starburst and the peak of the black hole accretion rate (BHAR) that is in agreement with observations (e.g., Wild et al. 2010). To check the agreement of our model with the $M_{\mathrm{BH}}-\sigma$ correlation (see, e.g., Di Matteo et al. 2005) we repeat the calculations for different initial galaxy masses. Figure 2 shows the mass of the black hole after the merging event, namely at the time the BHAR reaches its maximum value as function of the stellar velocity dispersion of the resulting elliptical galaxy. The results are consistent with the observed correlation. As the black hole continues growing after the galaxies have merged we additionally plot the range of black hole mass during this growth stage, namely from the time of the end of the starburst to the time the BHAR decreases to $5 \%$ of its maximum value. Our results suggest that this continuing evolution of the black hole mass may contribute to the large scatter of the observed $M_{\mathrm{BH}}-\sigma$ correlation.

\section{Summary}

With only one model setup we were able to reproduce three observational findings that have been identified in galaxies: (i) The observed time lag between starburst and AGN activity is principally caused by a viscous time lag the gas needs to flow through the AGN's accretion disk before reaching the central black hole. (ii) Our results match the observed $M_{\mathrm{BH}}-\sigma$ correlation. (iii) The large scatter of the $M_{\mathrm{BH}}-\sigma$ correlation is caused by the continuing evolution of the black hole mass after the merging event.

\section{References}

Debuhr, J., Quataert, E., \& Ma, C.-P. 2012, MNRAS 420, 2221

Di Matteo, T., Springel, V., \& Hernquist, L. 2005, Nature 433, 604 
Gültekin, K., Richstone, D. O., Gebhardt, K., Lauer, T. R., et al. 2009, ApJ 698, 198 Power, C., Nayakshin, S., \& King, A. 2011, MNRAS 412, 269

Scannapieco, C., Tissera, P. B., White, S. D. M., \& Springel, V. 2005, MNRAS 364, 552 Springel, V. 2005, MNRAS 364, 1105

Wild, V., Heckman, T., \& Charlot, S. 2010, MNRAS 405, 933 